\title{
Highly Sensitive, Ultra-Thin Dynamic Lateral Pressure Sensor Using Blue Laser Exposed Porous CNTs
}

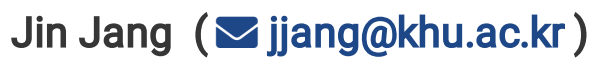

Kyung Hee University

Chanju Park

Kyung Hee University

Munsu Choi

Kyung Hee University

Suhui Lee

Kyung Hee University

Hyunho Kim

Kyung Hee University

Taeheon Lee

Kyung Hee University

M.M. Billah

Kyung Hee University

Byunglib Jung

Kyung Hee University

\section{Research Article}

Keywords:

Posted Date: February 1st, 2022

DOI: https://doi.org/10.21203/rs.3.rs-1298033/v1

License: (1) (1) This work is licensed under a Creative Commons Attribution 4.0 International License.

Read Full License 


\title{
Highly Sensitive, Ultra-Thin Dynamic Lateral Pressure Sensor Using Blue Laser Exposed Porous CNTs
}

\author{
Chanju Park, Munsu Choi, Suhui Lee, Hyunho Kim, Taeheon Lee, Mohammad \\ Masum Billah, Byunglib Jung and Jin Jang*
}

Advanced Display Research Center (ADRC), Department of Information Display, Kyung Hee University, 26, Kyungheedae-ro, Dongdaemun-gu, Seoul 02447, South Korea

*Corresponding author: Jin Jang (e-mail: jjang@khu.ac.kr).

\begin{abstract}
Piezoresistive sensor is an essential component of wearable electronics that can detect resistance changes when pressure is applied. An effective approach to enhance its performance is to make micro-structured device. In this paper, porous carbon nanotubes (CNTs) are formed by blue laser (BL) exposure on CNTs layer, which increases its thickness $\sim 4$ times compared to the as-deposited layer. Then, the pressure sensor is fabricated by spin coating of styrene-ethylene-butylene-styrene (SEBS) elastomer on the porous CNTs layer. A $1.32 \mu \mathrm{m}$ thick pressure sensor exhibits a high sensitivity of $6.54 \times 10^{6} \mathrm{kPa}^{-1}$, a wide sensing range of $278 \mathrm{~Pa} \sim 40 \mathrm{kPa}$, and fast response/recovery times of 900/760 $\mu$ s, respectively. The stability of the pressure sensor is demonstrated by repeated loading and unloading of $20 \mathrm{kPa}$ for 3600 cycles. The stretchable pressure sensor was also demonstrated using lateral CNT electrodes on SEBS surface, exhibiting stable pressure performance up to $20 \%$ stretching. Finally, a 32 x 32 active-matrix pressure sensor array is demonstrated consisting of amorphous $\mathrm{InGaZnO}_{4}$ thin-film transistor (TFT) backplane for pressure mapping and real-time monitoring. The sensor array demonstrates dynamic area touch by pen writing with $\sim 1 \mathrm{~cm} / \mathrm{s}$ speed.
\end{abstract}

\section{Introduction}

The pressure sensor is of increasing interest because of its various applications such as object detection, display touch, electronic skin (e-skin), etc. ${ }^{1-3}$ Mobile and healthcare devices with touch sensors are being used in human activities. ${ }^{4,5}$ The demand for the development of pressure sensors, converting the external stimulus into electrical signals, is continuously increasing. ${ }^{6-8}$ In general, the pressure sensors can be classified as piezoresistive, ${ }^{9-16}$ capacitive, ${ }^{17-26}$ and piezoelectric types. ${ }^{27-35}$

The piezoresistive type pressure sensors operate on a simple principle that detects the change in device resistance when pressure is applied. ${ }^{14-16}$ Usually, conductive fillers such as carbon nanotubes 
(CNTs), ${ }^{9,10,14}$ graphenes, ${ }^{11,36}$ metal nanoparticles, ${ }^{37-39}$ and nanowires ${ }^{16,40}$ have been used to fabricate piezoresistive type pressure sensors. However, polymer composite film-based piezoresistive pressure sensors have the disadvantages of low sensitivity. Therefore, the pressure sensors with porous sensing films, ${ }^{17,41,42}$ microstructure, ${ }^{13,43-46}$ and pyramid structure ${ }^{47-52}$ were developed to achieve improved sensitivity and response time. The microstructure efficiently modulates the contact resistance, improves the response speed, recovery speed, and stability of the pressure sensor. A pressure sensor with a sensitivity of $8655.6 \mathrm{kPa}^{-1}$ was fabricated through a pyramid microstructure by Huang et al., ${ }^{48}$ and another pressure sensor with a sensitivity of $83.9 \mathrm{kPa}^{-1}$ was fabricated from the porous structure by Zhao et al. ${ }^{41} \mathrm{In}$ general, the microstructure can be made by the complicated process, including molding and transfer processes. $^{47,53-55}$

In this study, porous CNT sensing layer was prepared by blue laser annealing (BLA) ${ }^{56}$ of conventional CNTs layer. Then, this porous CNT layer was mixed with styrene-ethylene-butylene-styrene (SEBS) elastomer to fabricate a stretchable pressure sensor. The fabricated pressure sensor exhibits a wide sensing range of $\sim 30 \mathrm{kPa}$ with a high sensitivity of $6.54 \times 10^{6} \mathrm{kPa}^{-1}$. It shows the fast-rising time of 900 $\mu$ s and falling time of $760 \mu \mathrm{s}$, with excellent mechanical durability against repeated loading and unloading test under high pressure of $20 \mathrm{kPa}$ for 3600 cycles. Finally, a-IGZO TFT-based 32 x 32 active-matrix pressure sensor array is demonstrated for pressure mapping and real-time monitoring with dynamic area touch capability by pen writing speed of $\sim 1 \mathrm{~cm} / \mathrm{s}$.

\section{Results and Discussion}

The schematic diagram of spray-coating of the CNTs and blue laser (BL) exposure on the CNTs layer to obtain porous layer are shown in Fig. 1a and 1b, respectively. The BL beam has a length of $520 \mu \mathrm{m}$ and $20 \mu \mathrm{m}$ width, as shown in Fig. 1c. The cross-sectional scanning electron microscopic (SEM) views of the CNTs before and after BL (laser energy density of $5.06 \mathrm{~J} \mathrm{~cm}^{-2}$ ) exposure are shown in Fig. 1d and 1e, respectively. The thickness of the as-sprayed CNTs layer is $330 \mathrm{~nm}$, which is uniform over the substrate and increases to $1.32 \mu \mathrm{m}$ upon BLA, which is almost 4 times by BL exposure. It is clearly observed that the surface morphology of CNTs layer changes significantly after BL exposure, where the top and bottom regions are very different, as shown in Fig. 1f and $1 \mathrm{~g}$, respectively. The morphology change of CNTs layer as a variation of incident BL energy density can be seen in Supplementary Fig. S1. With increasing the laser energy density up to $5.06 \mathrm{~J} \mathrm{~cm}^{-2}$, the top region of the CNTs layer changes its morphology to very porous structure due to the absorption of the BL beam. The carbon-carbon bond in CNTs structure breaks under laser exposure, and the broken $\mathrm{C}$ atoms aggregate with the other $\mathrm{C}$ atoms ${ }^{57}$ Note that the 
sensitivity of the piezoresistive sensor depends on the microstructure of the porous CNTs. ${ }^{44,58,59}$ The sheet resistance of BLA CNTs decreases with increasing BL exposure energy, as shown in Fig. 1h, from 955 ohm sq ${ }^{-1}$ to less than $490 \mathrm{ohm} \mathrm{sq}{ }^{-1}$ when the BL energy density increases to $5.06 \mathrm{~J} \mathrm{~cm}^{-2}$. The reduction in sheet resistance of the CNTs layer is mainly due to the evaporation of surfactants by the heat absorbed from BL energy and the sequential increase of electrical conductivity. ${ }^{60}$
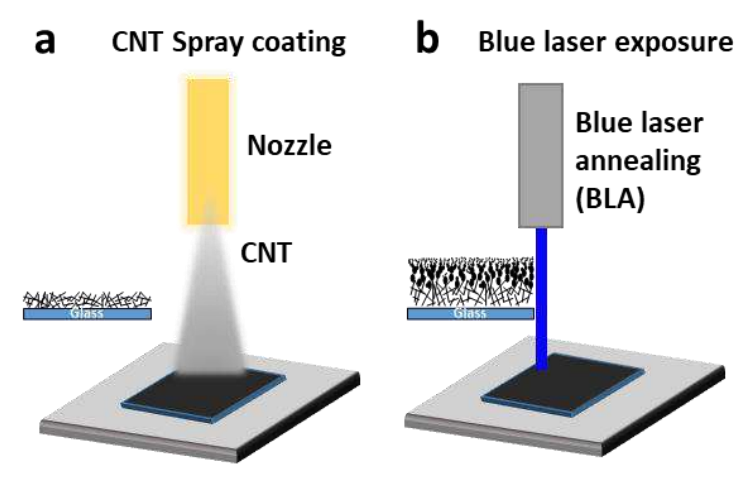

C BLA
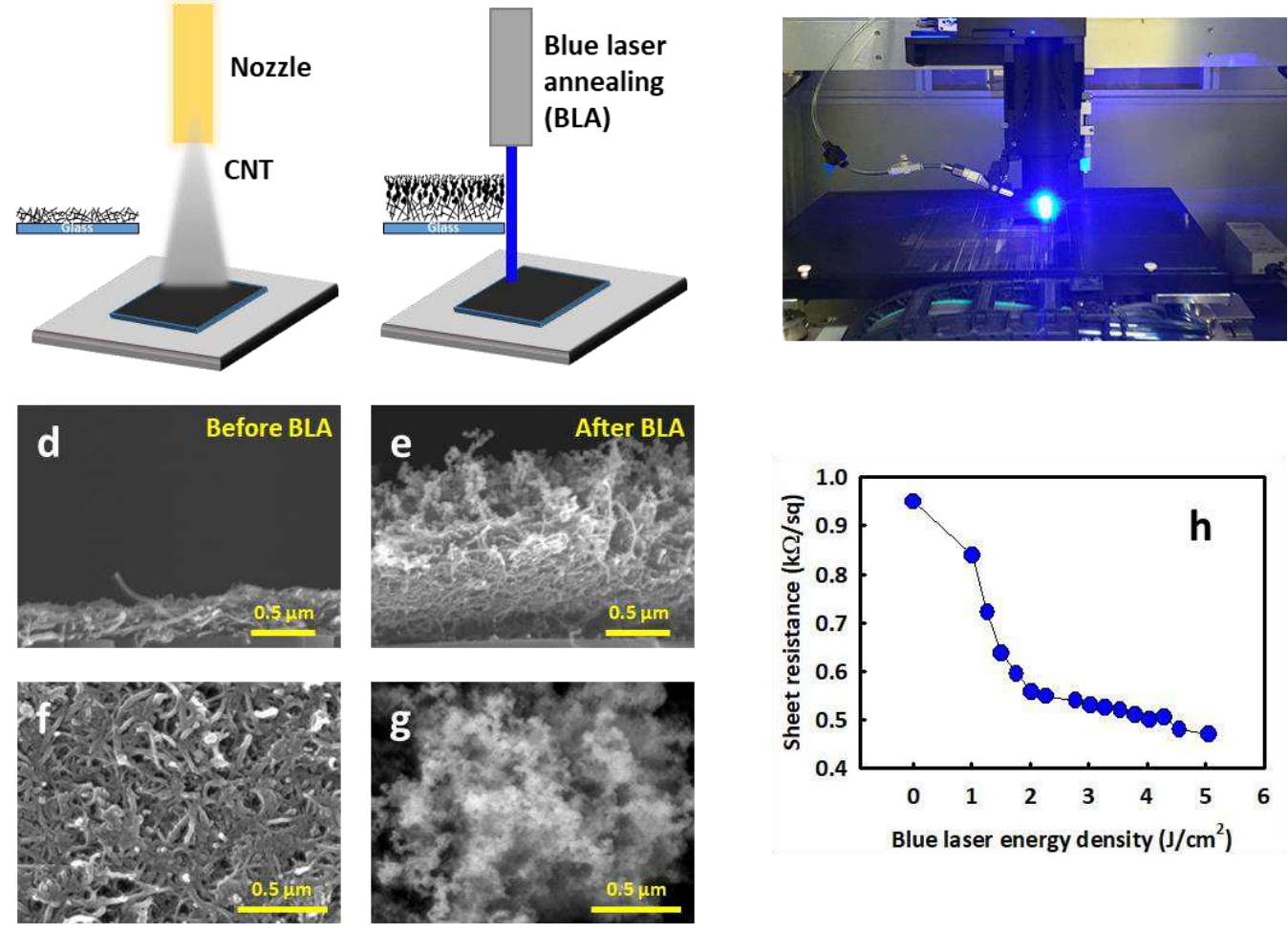

Fig. 1 Fabrication of porous CNTs by BL exposure, its morphology, and sheet resistance. Schematic views of a spray-coating of CNTs and b BLA on the CNTs layer. $\mathbf{c}$ BLA equipment with the BL beam of $520 \mu \mathrm{m}$ x $20 \mu \mathrm{m}$. d, e Cross-sectional and $\mathbf{f}, \mathbf{g}$ top SEM views of the CNTs layer before and after BLA (5.06 $\left.\mathrm{J} \mathrm{cm}^{-2}\right)$ respectively. $\mathbf{h}$ The sheet resistance of the porous CNTs as a function of BL energy density.

The porous CNTs film fabricated by BL exposure was coated with the highly elastic and stretchable SEBS layer to realize our pressure sensor. We considered SEBS as elastomeric matrix, of which the thickness could be controlled by changing the concentration diluted into toluene to achieve a highly sensitive, stretchable sensor. The detailed fabrication process of the porous CNT/SEBS pressure sensor is illustrated in Supplementary Fig. S2. The porous CNT/SEBS pressure sensor is simply fabricated by spincoating SEBS of $60 \mathrm{mg} \mathrm{ml}^{-1}$ on the porous CNTs layer of $2.5 \times 2.5 \mathrm{~cm}^{2}$. 
Fig. 2a shows the cross-sectional SEM image of porous CNT/SEBS composite, indicating that CNTs are filled with SEBS. The optical and SEM images of the porous CNT/SEBS layer are shown in Supplementary Fig. S3, indicating the CNTs with fully covered by SEBS elastomer. The CNT/SEBS film was detached from carrier glass as shown in Fig. $2 b$ and then transferred onto the lateral CNT electrodes on SEBS substrate. The fabrication process of stretchable pressure sensor was completed by the SEBS passivation, as shown in Fig. 2c. The detailed fabrication process of the stretchable pressure sensor can be seen in Supplementary Fig. S4. The schematic diagram for the operation of the pressure sensor is shown in Fig. 2d and 2e, with low and high pressures, respectively. Note that the top region of the CNT/SEBS film is more porous than the bottom CNT/SEBS layer due to more BL absorption at top region. Generally, the sensitivity of pressure sensor depends on the pressure range. At low-pressure region between $278 \mathrm{~Pa}$ to $537 \mathrm{~Pa}$, the current starts to flow between stretchable electrodes through porous CNTs/SEBS sensor through near bottom CNT region, as shown using yellow line direction in Fig. 2d. High sensitivity at low pressures can be achieved by causing a large resistance change at the porous CNTs. However, at high pressure, the currents are contributed from both top and bottom regions of the CNT/SEBS film and then gradually being saturated, leading to reduced sensitivity at high pressure region, as shown in Fig. 2e. The sensitivity of the proposed pressure sensor, therefore, depends on the pressure range.
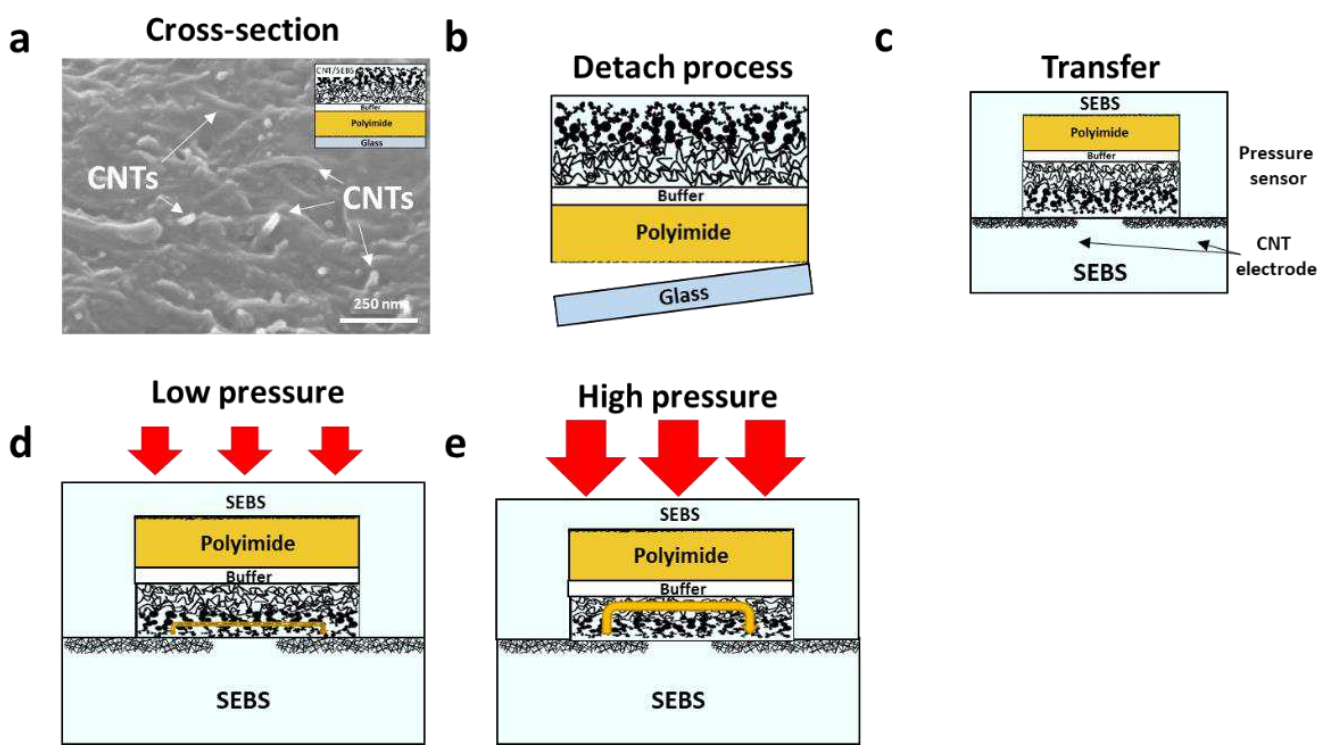

Fig. 2 The fabrication process of the stretchable pressure sensor and working principle under external pressure. a Cross-sectional SEM image of fabricated porous CNT/SEBS pressure sensor. Inset shows a cross-sectional schematic illustration of the pressure sensor. b Schematic of the mechanical detachment of the SEBS coated porous CNTs layer and $\mathbf{c}$ transfer of the CNTs sensor on the CNTs lateral electrodes on SEBS film. Mechanism of the pressure sensor: The schematic views of the operation of 
porous CNTs pressure sensor under $\mathbf{d}$ low external pressure $(0 \sim 0.5 \mathrm{kPa})$, and e high external pressure $(5$ $\sim 40 \mathrm{kPa}$ ). To characterize the piezoresistive performance, the piezoresistive response is monitored according to the applying pressure at a constant bias voltage, $\mathrm{V}=0.1 \mathrm{~V}$. The sensor performance using the porous CNT/SEBS layer under a variable pressure range at $278 \mathrm{~Pa} \sim 40 \mathrm{kPa}$ is shown in Supplementary Fig. S5. The measurements were done by loading and unloading using a force gauge on the CNT/SEBS sensor on glass substrate. The sensor shows a very low initial current (less than $\sim 10^{-11} \mathrm{~A}$ ) at zero pressure because of the existence of the insulting SEBS materials covered on the porous CNTs.

Fig. 3a shows the current changes plotted as a function of applied pressure. The sensitivity (S) is calculated by the following equation:

$S=\delta\left(\Delta I / I_{0}\right) / \delta P$,

where $\mathrm{I}_{0}$ is the initial current, $\Delta \mathrm{I}$ is the current change during the pressure loading, and $\mathrm{P}$ is applied pressure. The porous CNT/SEBS sensor shows a broad sensing range (278 $\mathrm{Pa} 40 \mathrm{kPa}$ ) with very high sensitivity of $1.13 \times 10^{5} \mathrm{kPa}^{-1}$ in low-pressure region $278 \sim 537 \mathrm{~Pa}, 6.54 \times 10^{6} \mathrm{kPa}^{-1}$ in medium pressure region $2 \sim 4 \mathrm{kPa}, 6.63 \times 10^{5} \mathrm{kPa}^{-1}$ in high-pressure region $5.7 \sim 38.9 \mathrm{kPa}$, respectively. High sensitivity can be achieved due to the extremely low initial current and highly sensitive current under applied external pressure. The comparison of pressure sensing performance without and with BLA on CNTs can be seen at the pressures of 2.5, 5.0, and $10 \mathrm{kPa}$, respectively, in Supplementary Fig. S6a and S6b. The sensor performance is also affected by the thickness of SEBS, as shown in Supplementary Fig. S7. When the thickness of SEBS layer is $2 \mu \mathrm{m}$, the sensor current is very low (less than $0.3 \mu \mathrm{A}$ ) at the high pressure of $150 \mathrm{kPa}$, but there is no response when the thickness of over $3 \mu \mathrm{m}$.

We compare the sensor performances and structures reported in the literatures shown in Table 1 together with our proposed device. Note that the thickness of our sensor is $1.32 \mu \mathrm{m}$ which is extremely thin compared to the others. Thinner sensors have many advantages, such as easy adoption in mobile electronic systems including displays.

Table 1. Summary of the performances of the pressure sensors with high sensitivity reported in the literatures.

\begin{tabular}{cccccc}
\hline Structure & $\begin{array}{c}\text { Pressure } \\
\text { range }(\mathrm{Pa})\end{array}$ & $\begin{array}{c}\text { Sensitivity } \\
\left(\mathrm{kPa}^{-1}\right)\end{array}$ & $\begin{array}{c}\text { Response/recovery } \\
\text { time }(\mathrm{ms})\end{array}$ & $\begin{array}{c}\text { Structure } \\
\text { thickness } \\
(\mu \mathrm{m})\end{array}$ & Reference \\
\hline $\begin{array}{c}\text { Polypyrrole/PDMS } \\
\text { micropyramid }\end{array}$ & $0.075-1 \mathrm{k}$ & $\begin{array}{c}1.9 \times 10^{3} \\
4.6 \times 10^{2}\end{array}$ & $0.05 / 6.2$ & $>20$ & 49 \\
$\begin{array}{c}\text { Sandpaper-molded } \\
\text { rGO/PDMS }\end{array}$ & $10-400 \mathrm{k}$ & $\begin{array}{c}2.5 \\
1.1 \times 10^{3}\end{array}$ & $150 / 40$ & $>500$ & 44 \\
$\begin{array}{c}\text { cellulose/nanowire } \\
\text { nanohybrid }\end{array}$ & $100-150 \mathrm{k}$ & $>5 \times 10^{3}$ & $-/<1$ & $\sim 1$ & 45
\end{tabular}




\begin{tabular}{|c|c|c|c|c|c|}
\hline $\begin{array}{c}\text { Au/PDMS } \\
\text { micropyramid \& } \\
\text { PEDOT:PSS/Au/PI }\end{array}$ & $0.425-2 \mathrm{k}$ & $\begin{array}{l}9.2 \times 10^{2} \\
1.2 \times 10^{4} \\
1.5 \times 10^{3}\end{array}$ & $0.44 / 0.08$ & - & 50 \\
\hline $\begin{array}{l}\text { Au/PMDS } \\
\text { pyramids }\end{array}$ & $0.25-56 \mathrm{k}$ & $\begin{array}{l}3.8 \times 10^{5} \\
2.7 \times 10^{5} \\
4.9 \times 10^{4}\end{array}$ & $75 / 50$ & 555 & 51 \\
\hline $\begin{array}{l}\text { PEDOT:PSS/PUD } \\
\text { interlocked with } \\
\text { TPU electrode }\end{array}$ & $\begin{array}{l}0.025- \\
100 \mathrm{k}\end{array}$ & $\sim 3.8 \times 10^{5}$ & $0.016 /-$ & $\sim 5$ & 46 \\
\hline $\begin{array}{c}\text { Ag nanocrystal } \\
\text { /PDMS janus-like } \\
\text { pyramid }\end{array}$ & $220-3 k$ & $\begin{array}{l}1.9 \times 10^{4} \\
1.6 \times 10^{6}\end{array}$ & - & $>250$ & 52 \\
\hline $\begin{array}{c}\text { Porous } \\
\text { CNT/SEBS }\end{array}$ & $278-40 \mathrm{k}$ & $\begin{array}{l}1.1 \times 10^{5} \\
6.5 \times 10^{6} \\
7.5 \times 10^{5}\end{array}$ & $<0.9 /<0.76$ & $\sim 1$ & This work \\
\hline
\end{tabular}

Fig. $3 \mathrm{~b}$ shows the real-time sensor performance at the pressure of $2.5 \sim 40 \mathrm{kPa}$. The measurements were performed with and without loading a pressure on the sensor. The result indicates that our sensor exhibits excellent sensing performance below $6 \mathrm{kPa}$, however, sensitivity saturates for above $6 \mathrm{kPa}$. Fig. 3c shows the response and recovery times of the pressure sensor. A faster response and recovery times of $900 \mu \mathrm{s}$ and $760 \mu \mathrm{s}$, respectively, explaining the excellent capability of the proposed pressure sensor. The mechanical durability of the sensors was tested by loading and unloading pressure of $20 \mathrm{kPa}$ at $\mathrm{V}=0.1 \mathrm{~V}$ for 4000 cycles. Fig. $3 d$ shows stable current changes with a high current ratio even at high pressure of 20 $\mathrm{kPa}$ until 4000 cycles. 
a

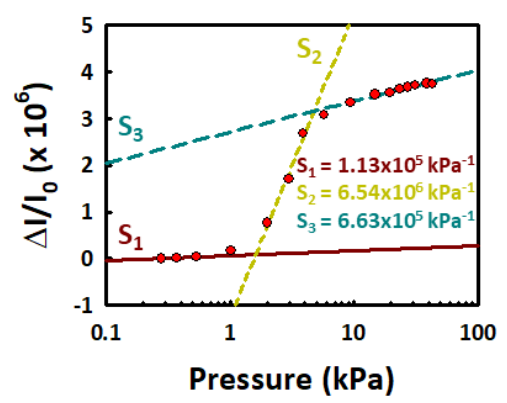

C

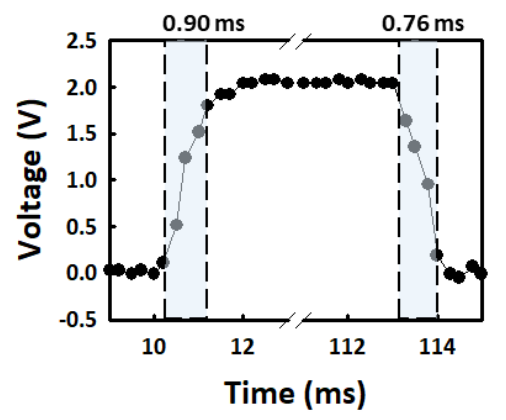

b

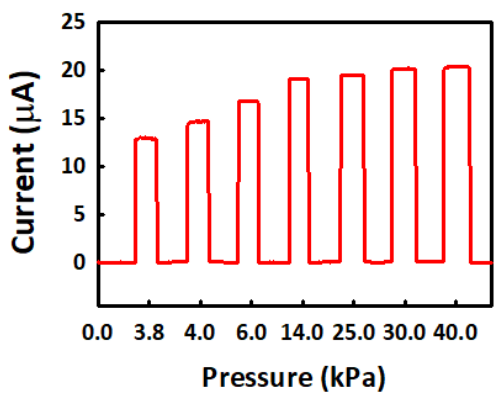

d

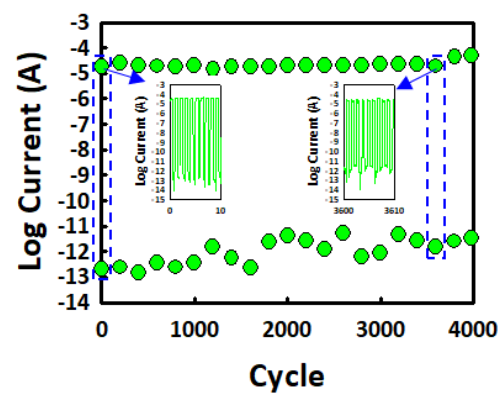

Fig. 3 Electrical characteristics of porous CNT/SEBS pressure sensor. a Relative current change as a function of pressure at V $=0.1 \mathrm{~V}$. Sensitivities (S1, S2, S3) were extracted in low-, middle-, high-pressure regions. b Real-time measurements of current changes under different pressures. c The response and recovery times of the pressure sensor. d Durability test of the pressure sensor until 4000 cycles at $20 \mathrm{kPa}$.

Fig. $4 \mathrm{a}$ and $4 \mathrm{~b}$ shows the photographs of stretchable pressure sensor at $20 \%$ stretched and released states, respectively, using a stretchability measurement machine. ${ }^{61}$ Fig. $4 \mathrm{c}$ shows the current change of the pressure sensor with and without strain under dynamic pressure. Note that the stretchability of pressure sensor could be achieved by fabricating the pressure sensor on rigid and flexible polyimide (PI) island transferred onto SEBS substrate. Fig. 4d shows the relative current changes of the pressure sensor plotted as a function of strain. It demonstrates that the stretchable pressure sensor can be operated well at $20 \%$ strain. The stretchable pressure sensor was mounted to the joint of a finger to mimic the motions of the human body as shown in Fig. 4e and $4 \mathrm{f}$ for the real-time current measurement as shown in Fig. 4g. Fig. 4h shows the current changes in the stretchable pressure sensor under different bending angles. The results show that the current increases and reaches to the maximum at $60^{\circ}$. A bending angle above $60^{\circ}$ leads to a reduction of sensing current between CNT electrodes, which might be due to the reduction of sensor thickness of CNT/SEBS film. 

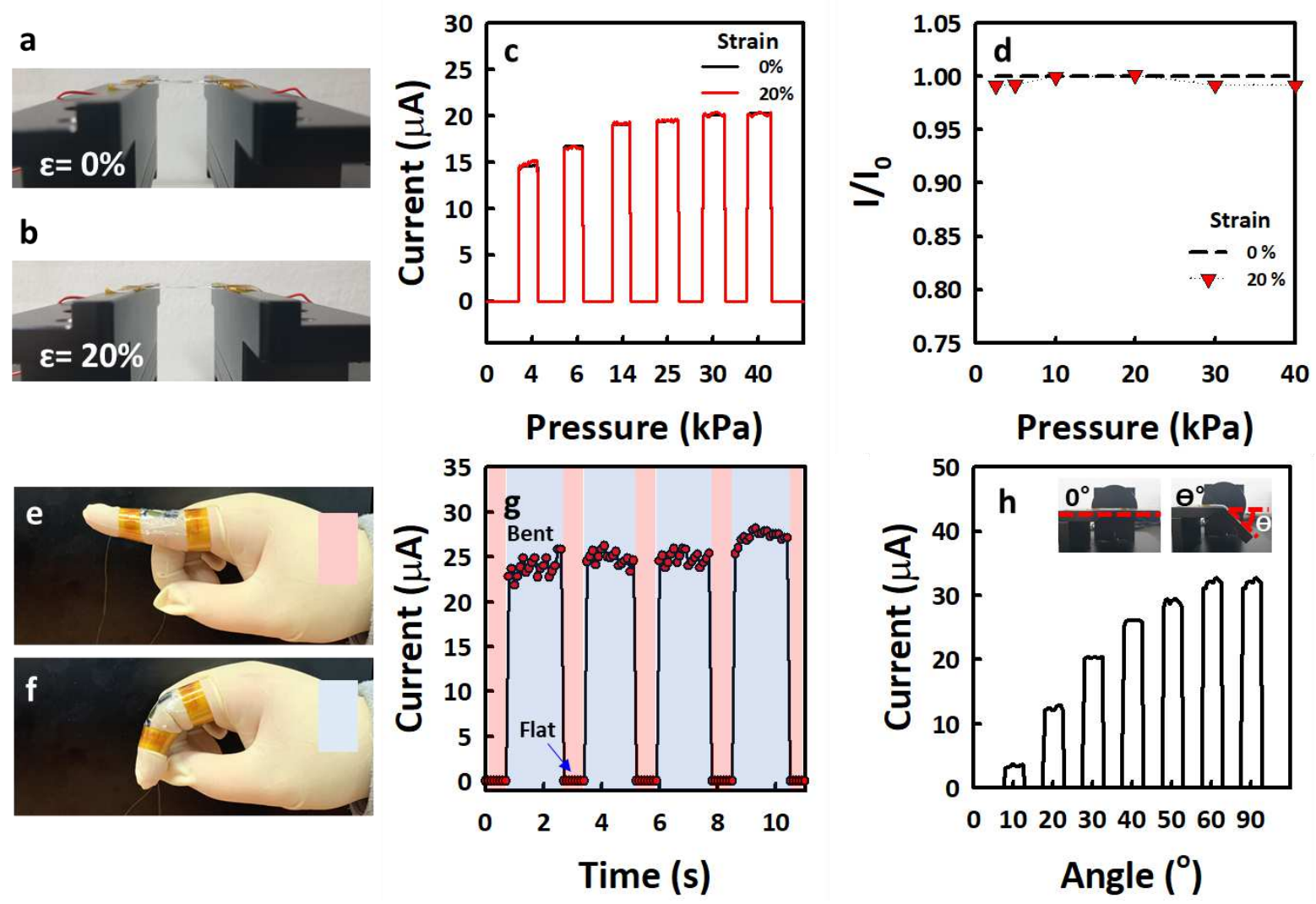

Fig. 4 Performance of the stretchable porous CNTs pressure sensor. Photographs of a stretchable pressure sensor under a $0 \%$ and b $20 \%$ strain. c Current change of the stretchable pressure sensor by loading a copper block under $0 \%$ and $20 \%$ stretching, respectively. d Normalized currents as a function of external pressure measured at $0 \%$ and $20 \%$ strain, respectively. $\mathrm{I}_{0}$ is the initial current before stretching, and $I$ is the current measured after applying an external strain of $20 \%$ stretching. Photographs of stretchable pressure sensor attached on the finger at $\mathbf{e}$ flat and $\mathbf{f}$ bent state. $\mathbf{g}$ The current change of the stretchable pressure sensor on finger under flat state (red) and bent state (blue). $\mathbf{h}$ The current change of the stretchable pressure sensor under bending of the angle between 0 and $90^{\circ}$.

The porous CNT/SEBS film was integrated with amorphous $\mathrm{InGaZnO}_{4}$ (a-IGZO) thin-film transistor (TFT) backplane for a high-resolution active-matrix sensor array. Fig. 5a,b shows the photograph and cross-sectional illustration of a 32 × 32 active-matrix pressure sensor array using the CNT/SEBS film on the backplane. The detailed process flow of a-IGZO TFT can be seen in the Method section. The CNT/SEBS film was flipped and transferred on the a-IGZO TFT backplane. The CNT/SEBS film, pressure sensor, makes contact through the two vias with the patterned electrodes metal layer in a pixel. Fig. 5c shows a schematic of a pixel circuit, which consists of three switching TFTs, one $1 \mathrm{pF}$ capacitor, and one pressure sensor. The optical image of active-matrix (AM) sensor array can be seen in Fig. 5d, and 
the one-pixel dimension is $626 \times 625 \mu \mathrm{m}^{2}$. Fig. 5c shows a schematic of a pixel circuit, which consists of three switching TFTs, one $1 \mathrm{pF}$ capacitor, and one pressure sensor. The optical image of active-matrix (AM) sensor array can be seen in Fig. 5d, and the one-pixel dimension is 626 x $625 \mu \mathrm{m}^{2}$, and the contact area between the metal electrode and sensor is $30 \mathrm{x} 400 \mu \mathrm{m}^{2}$. Fig. 5e and $\mathrm{f}$ show transfer and output characteristics of a-IGZO TFT $(\mathrm{W} / \mathrm{L}=20 / 6 \mu \mathrm{m})$ in the pixel circuit. The threshold voltage $\left(\mathrm{V}_{\text {th }}\right)$, fieldeffect mobility $\left(\mu_{\mathrm{fe}}\right)$, and subthreshold swing (SS) of the a-IGZO TFT are $-0.3 \mathrm{~V}, 18.6 \mathrm{~cm}^{2} / \mathrm{Vs}, 0.53 \mathrm{~V} / \mathrm{dec}$, respectively. All the TFTs were used as switching units for charging, readout, and reset periods. Fig. $5 \mathrm{~g}$ shows the timing diagram of the pixel circuit. The concept of readout circuit is to read the charges stored at the q node for the resistance change during the pressure being applied. The operation of the pixel, such as pre-charging, readout, and reset of the pixel circuit, is shown in Supplementary Fig. S8. In the precharging state, the gate signal from the $n-1^{\text {th }}$ stage is applied to T1, as shown in Supplementary Fig. S8a. Then, $\mathrm{T} 1$ is turned on, and the $\mathrm{q}$ node could be charged depending on the resistance value. The CNT/SEBS sensor can demonstrate a wide sensing range from $278 \mathrm{~Pa}$ to $40 \mathrm{kPa}$, corresponding current change from $2.5 \mathrm{nA}$ to $20 \mu \mathrm{A}$ as shown in Fig. 3a. Note that the off-state current of the sensor is less than 10 pA, as shown in Supplementary Fig. S5. When T2 and T3 are turned off, and capacitor is serially connected to the q node, charged voltage at q node $\left(\mathrm{V}_{\mathrm{q}}\right)$ could be obtained from the following equation :

$\mathrm{V}_{\mathrm{q}}=\mathrm{V}_{\mathrm{DD}} *\left(1-\mathrm{e}^{(-\mathrm{t} / \mathrm{RC})}\right)$,

where $\mathrm{Vq}, \mathrm{V}_{\mathrm{DD}}, \mathrm{t}, \mathrm{R}$, and $\mathrm{C}$ are charged voltage at $\mathrm{q}$ node, input voltage, gate pulse width, the resistance of pressure sensor, and capacitance of $1 \mathrm{pF}$, respectively. The $\mathrm{V}_{\mathrm{q}}$ is decided by the $\mathrm{n}-\mathrm{1}^{\text {th }}$ scan signal time $\mathrm{t}$ and the resistance of pressure sensor R. We set all scan signal time to be $20 \mu$ s for detecting the lowpressure sensing region (278 $\mathrm{Pa}(39.7 \mathrm{M}$-ohm) to $537 \mathrm{~Pa}(0.61 \mathrm{M}$-ohm)). When the resistance of the pressure sensor is between 0.61 and $39.7 \mathrm{M}$-ohm, it is called the low-pressure region in this paper. The RC time, $3 \tau$, reaching $95 \%$ of $\mathrm{V}_{\mathrm{DD}}$, would be $1.82 \mu \mathrm{s}$ and $119 \mu \mathrm{s}$, respectively. This means that $\mathrm{V}_{\mathrm{q}}$ could be determined by scan time, $\mathrm{t}$. If the scan time $\mathrm{t}$ is $20 \mu \mathrm{s}, \mathrm{V}_{\mathrm{q}}$ would be 0.395 x $\mathrm{V}_{\mathrm{DD}}$ under $278 \mathrm{~Pa}(39.7 \mathrm{M}$ ohm) and fully charged under $537 \mathrm{~Pa}\left(0.61 \mathrm{M}\right.$-ohm). The charges will go through the $\mathrm{V}_{\text {Out }}$ node in Supplementary Fig. S8b. Then, the $n+1^{\text {th }}$ scan signal is applied to turn on T3, and the q node is discharged to $\mathrm{V}_{\mathrm{SS}}$, as shown in Supplementary Fig S8c. The simulation results using Smart Spice are shown in Fig. $5 \mathrm{~h}$ when the scan signal of $10 \mathrm{~V}$ is applied to gate for $20 \mu \mathrm{s}$. The charges in the q node depend on the resistance of pressure sensor.

The gate driver was integrated into the sensor array. The gate shift register (GSR) was designed and used to apply the gate pulse to the pixels, as shown in Supplementary Fig. S9. Three scan signals are connected to one pixel, as shown in Supplementary Fig. S9a. Two sequential scan lines are applied to a particular pixel. The nth scan line is divided into two parallel lines at the nth stage and connected to the particular 
pixel and is also connected to the $n-1^{\text {th }}$ and $n+1^{\text {th }}$ pixels. The timing diagram and optical image of GSR are shown respectively in Supplementary Fig. S9b and S9c. The detailed operation of GSR appears in our previous work. ${ }^{62}$ The GSR output signals are working through the last stage, $32^{\text {nd }}$, as can be seen in Supplementary Fig. S9d. The pressure mapping was tested using the capital letters "A", "D", "R", and "C". The 32 x 32 sensor array shows the clear pressure response without crosstalk, as shown in Fig. 5i-l. The $\mathrm{V}_{\mathrm{DD}}, \mathrm{V}_{\mathrm{GS}}$, and $\mathrm{t}$ used for the sensor operation are $2.84 \mathrm{~V}, 10 \mathrm{~V}$, and $20 \mu \mathrm{s}$, respectively. The letters are softly pressed under $300 \mathrm{~Pa}$ to detect the characters in the low-pressure sensing region. The output signals of the pressed region are $1.32 \mathrm{~V}$, which is similar to the simulation value of $1.33 \mathrm{~V}$.

We also demonstrated the dynamic pressure distribution for real-time monitoring. The experimental set for displaying dynamic sensing on the screen is shown in Supplementary Fig. S10. The circuit schematic for the integrated AM sensor array can be seen in Supplementary Fig. S10a. The photographs of the customized board and zig with full integration are shown in Supplementary Fig. S10b and S10c. Using the driving board, the pressure detection can be seen in the range as shown in Video VS1, demonstrating the sensing Alphabet (A, D, R, C) writing on the sensor array respectively. Table 2 shows the summary of AM pressure sensor arrays reported in the literatures. Previous studies on the AM pressure sensors use the sensors such as commercial products (Pressure-sensitive rubber, PSR), ${ }^{63-70}$ and conductive filler/polymer composites, ${ }^{2}$ and pressure-sensitive TFT. ${ }^{4,71}$ The key advantage of the sensor is ultra-thin $(1 \mu \mathrm{m})$ so that can be applied to large-area electronic skin. The real-time writing on the AM sensor array can be seen on the PC screen. 
a
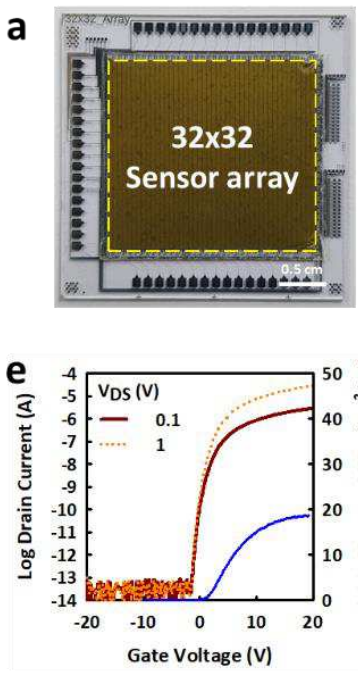

i

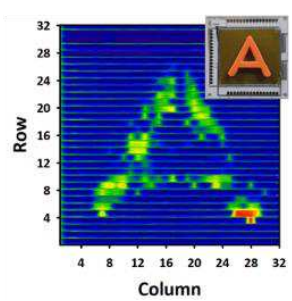

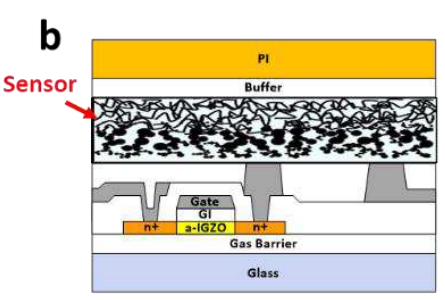
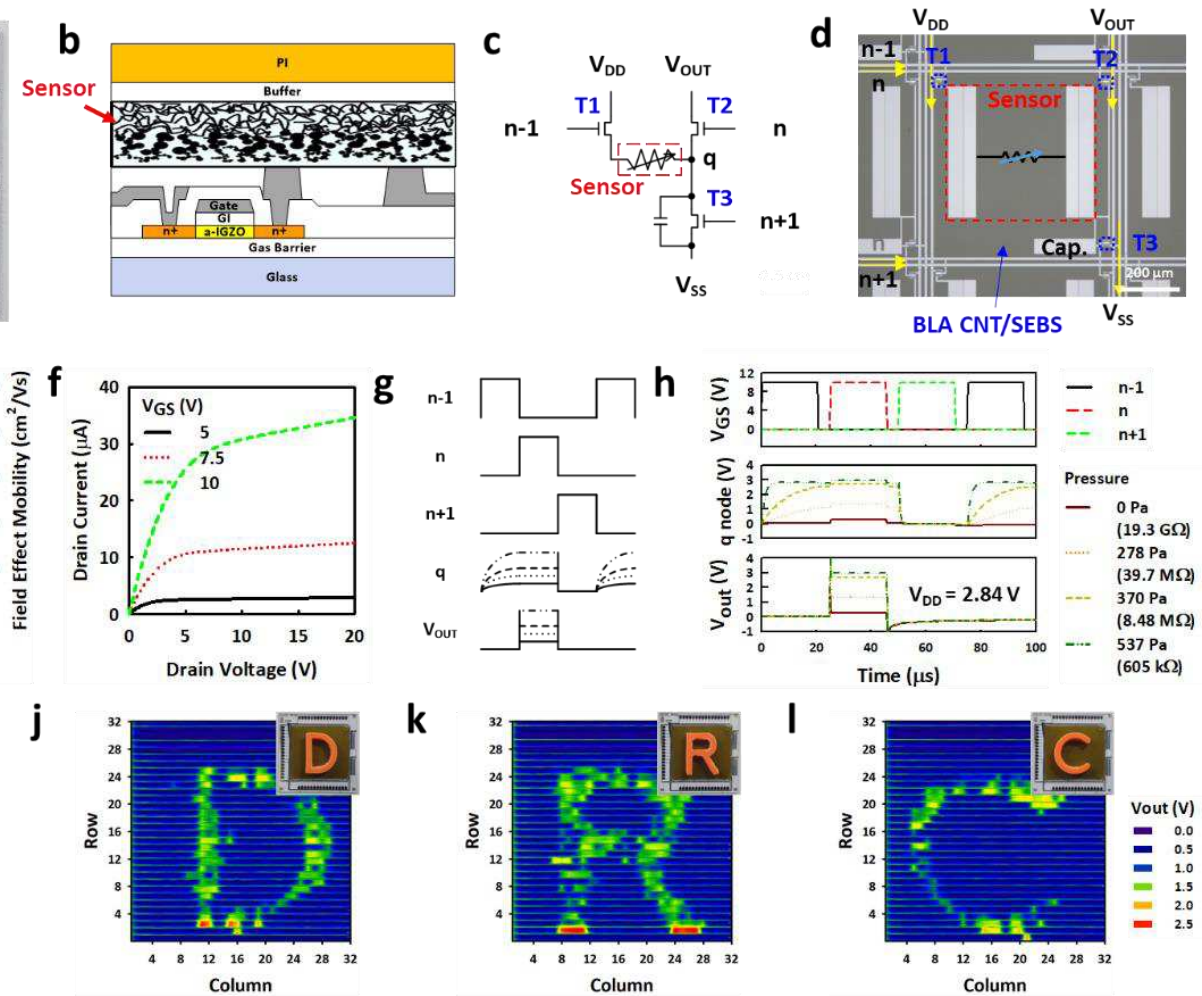

Fig. 5 Pressure mapping on the integrated 32 × 32 a-IGZO active-matrix sensor array. a optical image of the active-matrix pressure sensor array with BLA CNT/SEBS film. b Schematic illustration of AM sensor array. $\mathbf{c}$ schematic and $\mathbf{d}$ back optical image of a pixel circuit. One pixel consists of three TFTs, one capacitor, and one pressure sensor. Red dashed box shows current flowing area contacted with the lateral electrode. $\mathbf{e}$ Transfer and $\mathbf{f}$ output characteristics a-IGZO TFT. $\mathbf{g}$ The timing diagram of a pixel circuit and $\mathbf{h}$ simulated results with pressure variation $(0 \sim 537 \mathrm{~Pa})$. Pressure mapping of fabricated $32 \mathrm{x}$ 32 AM pressure sensor with letters i A, j D, k R, and l C, respectively. Inset shows letters placed on the sensor array.

Table 2. Summary of the reported active-matrix pressure sensor arrays in the literatures.

\begin{tabular}{|c|c|c|c|c|c|c|}
\hline $\begin{array}{l}\text { Pressure } \\
\text { sensor }\end{array}$ & $\begin{array}{c}\text { TFT (W/L, } \\
\mu \mathrm{m})\end{array}$ & $\begin{array}{l}\text { Driving } \\
\text { voltage } \\
\left(\mathrm{V}_{\mathrm{GS}} / \mathrm{V}_{\mathrm{DS}}\right.\end{array}$ & Resolution & Pixel size & $\begin{array}{l}\text { Output } \\
\text { signal }\end{array}$ & Reference \\
\hline PSR & $\begin{array}{c}\mathrm{Ge} / \mathrm{Si} \mathrm{NW} \\
(250 / 3)\end{array}$ & $5 / 0.5$ & $19 \times 18$ & $2.5 \mathrm{~mm}$ & $\sim 15 \mathrm{kPa}$ & 63 \\
\hline PSR & $\begin{array}{c}\text { CNT } \\
(2000 / 20)\end{array}$ & $-5 / 10$ & $16 \times 16$ & $\sim 1 \mathrm{~mm}$ & $\begin{array}{l}600 \mu \mathrm{A} \\
\text { at } 39.2 \mathrm{kPa}\end{array}$ & 64 \\
\hline PSR & $\begin{array}{c}\text { SWCNT } \\
(1250 / 100)\end{array}$ & $-10 /-5$ & $20 \times 20$ & - & $\begin{array}{c}\sim 1 \mu \mathrm{A} \\
\text { at } 7.2 \mathrm{kPa}\end{array}$ & 65 \\
\hline PSR & $\begin{array}{c}\text { SWCNT } \\
(365 / 130)\end{array}$ & $-20 /-20$ & $20 \times 20$ & 9.3 ppi & $\begin{array}{l}\sim 0.5 \mu \mathrm{A} \\
\text { at } 17.8 \mathrm{kPa}\end{array}$ & 66 \\
\hline
\end{tabular}




\begin{tabular}{|c|c|c|c|c|c|c|}
\hline PSR & $\begin{array}{c}\text { CNT } \\
(75 / 20)\end{array}$ & $-3 /-1$ & $16 \times 16$ & $4 \mathrm{~mm}$ & $\begin{array}{c}\sim 2.5 \mu \mathrm{A} \\
\text { at } 6.8 \mathrm{kPa}\end{array}$ & 67 \\
\hline PSR & $\begin{array}{l}\text { Pentacene } \\
(2800 / 120)\end{array}$ & $-20 /-20$ & $16 \times 16$ & $2.54 \mathrm{~mm}$ & $\sim-10 \mu \mathrm{A}$ & 68 \\
\hline PSR & $\begin{array}{l}\text { Pentacene } \\
(1800 / 50)\end{array}$ & $-40 /-40$ & $12 \times 12$ & $4 \mathrm{~mm}$ & $\begin{array}{c}6.7 \mu \mathrm{A} \\
\text { at } 30 \mathrm{kPa}\end{array}$ & 69 \\
\hline PSR & $\begin{array}{l}\text { Pentacene } \\
(2500 / 150)\end{array}$ & $-40 /-40$ & $7 \times 10$ & $\sim 5 \mathrm{~mm}$ & $\begin{array}{c}-0.3 \mu \mathrm{A} \\
\text { at } 30 \mathrm{kPa}\end{array}$ & 70 \\
\hline $\begin{array}{c}\text { CNT/graphene } \\
\text { nanofiber } \\
\text { sheet }\end{array}$ & $\begin{array}{c}\text { DNTT } \\
(\sim 5000 / \sim 50)\end{array}$ & $-10 /-10$ & $12 \times 12$ & - & $\begin{array}{l}>20 \mu \mathrm{A} \\
\text { at } 1 \mathrm{kPa}\end{array}$ & 2 \\
\hline $\begin{array}{l}\text { Pressure- } \\
\text { sensitive } \\
\text { OTFT }\end{array}$ & $\begin{array}{c}\text { PiI2T-Si } \\
(11520 / 40)\end{array}$ & $-100 /-100$ & $4 \times 4$ & $\sim 1.7 \mathrm{~mm}^{2}$ & $\begin{array}{c}>10 \mu \mathrm{A} \\
\text { at } 11 \mathrm{kPa}\end{array}$ & 4 \\
\hline $\begin{array}{l}\text { Suspended } \\
\text { gate }\end{array}$ & $\begin{array}{c}\text { PDPP3T } \\
(8500 / 80)\end{array}$ & $-60 /-60$ & $8 \times 8$ & $\sim 2.5 \mathrm{~mm}^{2}$ & $\begin{array}{l}-12 \mu \mathrm{A} \\
\text { at } 1.2 \mathrm{kPa}\end{array}$ & 71 \\
\hline $\begin{array}{c}\text { BLA } \\
\text { CNT/SEBS }\end{array}$ & $\begin{array}{c}\text { A-IGZO } \\
(20 / 6)\end{array}$ & $10 / 2.84$ & $32 \times 32$ & $625 \mu \mathrm{m}$ & $\begin{array}{c}20 \mu \mathrm{A} \\
\text { at } 20 \mathrm{kPa}\end{array}$ & $\begin{array}{l}\text { This } \\
\text { work }\end{array}$ \\
\hline
\end{tabular}

\section{Conclusion}

In summary, a highly sensitive pressure sensor is demonstrated using porous CNTs by the BL exposure on the CNTs layer. The CNTs were coated with SEBS elastomer, resulting in a very thin $(1.32 \mu \mathrm{m})$ highly sensitive pressure sensor. The sensor is capable to operate for the wide pressure range from $278 \mathrm{~Pa}$ to 40 $\mathrm{kPa}$. The pressure sensor shows the high sensitivity of $6.54 \times 10^{6} \mathrm{kPa}^{-1}$ at pressure range of $2 \mathrm{kPa} \sim 3.89$ $\mathrm{kPa}$ and high current ratio of $>400$ at the lowest pressure of $278 \mathrm{~Pa}$. The pressure sensor has the fastrising time of $900 \mu \mathrm{s}$ and falling-time of $760 \mu$ s and excellent durability with repeated loading and unloading tests under $20 \mathrm{kPa}$ for 3600 cycles. It maintains its piezoresistive characteristic until $20 \%$ stretching when it was made with CNTs lateral electrodes on SEBS substrate. The porous CNT/SEBS film was integrated on a $32 \times 32$ oxide TFT array to display the letters and dynamic writing. Therefore, porous CNT/SEBS film on oxide TFT array can be applied to ultra-thin large area e-skin sensors.

\section{Methods}

Fabrication of porous CNT/SEBS film. The process flow of CNTs coating and BL exposure on the CNTs layer to make porous CNTs can be seen in Supplementary Fig. S2. A CNTs/graphene oxide (GO) layer was spray-coated on the carrier glass using a mixture of CNT/GO solution using the mixing ratio of $\mathrm{CNT} / \mathrm{GO}=1 / 8$ for detaching the PI substrate from the carrier glass. The very thin CNT/GO layer was soft-baked at $130{ }^{\circ} \mathrm{C}$ for $15 \mathrm{~min}$ in air and then hard-baked at $290{ }^{\circ} \mathrm{C}$ for $2 \mathrm{~h}$ in a vacuum oven. The PI 
layer was then spin-coated on glass and then soft-baked at $\sim 140{ }^{\circ} \mathrm{C}$ for $30 \mathrm{~min}$ in a hot plate and then cured at $450{ }^{\circ} \mathrm{C}$ for $2 \mathrm{~h}$ in $\mathrm{N}_{2}$ atmosphere. A buffer layer of $\mathrm{SiNx} / \mathrm{SiO}_{2}$ was deposited on the PI substrate at the substrate temperature of $420{ }^{\circ} \mathrm{C} .{ }^{72,73}$ The surface of the $\mathrm{SiO}_{2}$ layer was treated with $\mathrm{UV} / \mathrm{O}_{3}$ for $300 \mathrm{~s}$ for uniform coating of CNTs by spray on the carrier glass and annealed at $290{ }^{\circ} \mathrm{C}$, for $2 \mathrm{~h}$ in a vacuum. The CNTs layer was exposed by a line beam of a BL (beam size: $520 \mu \mathrm{m}$ x $20 \mu \mathrm{m}$, laser energy density $5.06 \mathrm{~J} \mathrm{~cm}^{-2}$ ) as shown in Supplementary Fig. S1c. The $60 \mathrm{mg} \mathrm{ml}^{-1}$ SEBS solution diluted in toluene was spin-coated and cured on BLA CNT at $120^{\circ} \mathrm{C}$ for 10 minutes.

Fabrication of a-IGZO TFT backplane. On the buffer layer, $30 \mathrm{~nm}$ a-IGZO was deposited by reactive sputtering using a polycrystalline IGZO target $\left(\mathrm{InO}_{3}: \mathrm{Ga}_{2} \mathrm{O}_{3}: \mathrm{ZnO}=1: 1: 1 \mathrm{~mol} \%\right)$. Then, a $100 \mathrm{~nm}$-thick $\mathrm{SiO}_{2}$ was deposited on the top of the a-IGZO by PECVD as a gate insulator (GI) without breaking vacuum. A $100 \mathrm{~nm}$ Mo was deposited by sputtering and patterned as the top gate electrode. GI layer was etched by a self-aligned process with the gate pattern. Then, a $300 \mathrm{~nm}$-thick $\mathrm{SiO}_{2}$ layer was deposited as the interlayer by PECVD followed by the formation of via holes, and a $200 \mathrm{~nm}$-thick Mo layer was deposited and patterned for the $\mathrm{S} / \mathrm{D}$ electrodes. Then, $\mathrm{SiN}_{\mathrm{x}}$ and $\mathrm{SiO}_{2}$ double passivation layers were deposited through PECVD at $200{ }^{\circ} \mathrm{C}$. Finally, the devices were annealed at $300{ }^{\circ} \mathrm{C}$ in a vacuum for $1 \mathrm{~h}$.

\section{References}

1. Pan, L. et al. An ultra-sensitive resistive pressure sensor based on hollow-sphere microstructure induced elasticity in conducting polymer film. Nat. Commun. 5, 3002 (2014).

2. Lee, S. et al. A transparent bending-insensitive pressure sensor. Nat. Nanotechnol. 11, 472 (2016).

3. Wang, X. et al. Recent Progress in Electronic Skin. Adv. Sci. 2, 1-21 (2015).

4. Schwartz, G. et al. Flexible polymer transistors with high pressure sensitivity for application in electronic skin and health monitoring. Nat. Commun. 4, 1859 (2013).

5. Tao, L. Q. et al. Graphene-Paper Pressure Sensor for Detecting Human Motions. ACS Nano 11, 8790-8795 (2017).

6. Zhao, S. et al. Recent Advancements in Flexible and Stretchable Electrodes for Electromechanical Sensors: Strategies, Materials, and Features. ACS Appl. Mater. Interfaces 9, 12147-12164 (2017).

7. Choi, S., Lee, H., Ghaffari, R., Hyeon, T. \& Kim, D. H. Recent Advances in Flexible and Stretchable Bio-Electronic Devices Integrated with Nanomaterials. Adv. Mater. 28, 4203-4218 (2016).

8. Trung, T. Q. \& Lee, N. E. Recent Progress on Stretchable Electronic Devices with Intrinsically Stretchable Components. Adv. Mater. 29, 1603167 (2017).

9. Chou, H. H. et al. A chameleon-inspired stretchable electronic skin with interactive colour changing controlled by tactile sensing. Nat. Commun. 6, 8011 (2015). 
10. Bae, G. Y. et al. Pressure/Temperature Sensing Bimodal Electronic Skin with Stimulus Discriminability and Linear Sensitivity. Adv. Mater. 30, 1803388 (2018).

11. Zhao, L. et al. Construction of sandwich-like porous structure of graphene-coated foam composites for ultrasensitive and flexible pressure sensors. Nanoscale 11, 10229-10238 (2019).

12. Jian, M. et al. Flexible and Highly Sensitive Pressure Sensors Based on Bionic Hierarchical Structures. Adv. Funct. Mater. 27, 1606066 (2017).

13. Su, B., Gong, S., Ma, Z., Yap, L. W. \& Cheng, W. Mimosa-inspired design of a flexible pressure sensor with touch sensitivity. Small 11, 1886-1891 (2015).

14. Kim, K. H. et al. Wearable Resistive Pressure Sensor Based on Highly Flexible Carbon Composite Conductors with Irregular Surface Morphology. ACS Appl. Mater. Interfaces 9, 17499-17507 (2017).

15. Nie, P. et al. High-Performance Piezoresistive Electronic Skin with Bionic Hierarchical Microstructure and Microcracks. ACS Appl. Mater. Interfaces 9, 14911-14919 (2017).

16. Gong, S. et al. A wearable and highly sensitive pressure sensor with ultrathin gold nanowires. Nat. Commun. 5, 3132 (2014).

17. Kang, S. et al. Highly Sensitive Pressure Sensor Based on Bioinspired Porous Structure for RealTime Tactile Sensing. Adv. Electron. Mater. 2, 1600356 (2016).

18. Kwon, D. et al. Highly Sensitive, Flexible, and Wearable Pressure Sensor Based on a Giant Piezocapacitive Effect of Three-Dimensional Microporous Elastomeric Dielectric Layer. ACS Appl. Mater. Interfaces 8, 16922-16931 (2016).

19. Pruvost, M., Smit, W. J., Monteux, C., Poulin, P. \& Colin, A. Polymeric foams for flexible and highly sensitive low-pressure capacitive sensors. npj Flex. Electron. 3, 13-18 (2019).

20. Li, T. et al. Flexible Capacitive Tactile Sensor Based on Micropatterned Dielectric Layer. Small 12, 5042-5048 (2016).

21. You, B., Han, C. J., Kim, Y., Ju, B. K. \& Kim, J. W. A wearable piezocapacitive pressure sensor with a single layer of silver nanowire-based elastomeric composite electrodes. J. Mater. Chem. A 4, 10435-10443 (2016).

22. Kim, S. Y., Jee, E., Kim, J. S. \& Kim, D. H. Conformable and ionic textiles using sheath-core carbon nanotube microyarns for highly sensitive and reliable pressure sensors. $R S C A d v$. 7, 23820-23826 (2017).

23. Kim, S. Y. et al. Highly Sensitive and Multimodal All-Carbon Skin Sensors Capable of Simultaneously Detecting Tactile and Biological Stimuli. Adv. Mater. 27, 4178-4185 (2015).

24. Kou, H. et al. Wireless wide-range pressure sensor based on graphene/PDMS sponge for tactile monitoring. Sci. Rep. 9, 1-7 (2019).

25. Wang, J. et al. A highly sensitive and flexible pressure sensor with electrodes and elastomeric interlayer containing silver nanowires. Nanoscale 7, 2926-2932 (2015).

26. Boutry, C. M. et al. A Sensitive and Biodegradable Pressure Sensor Array for Cardiovascular Monitoring. Adv. Mater. 27, 6954-6961 (2015). 
27. Geng, D., Han, S., Seo, H., Mativenga, M. \& Jang, J. Piezoelectric Pressure Sensing Device Using Top-Gate Effect of Dual-Gate a-IGZO TFT. IEEE Sens. J. 17, 585-586 (2017).

28. Kim, D. Il et al. A sensor array using multi-functional field-effect transistors with ultrahigh sensitivity and precision for bio-monitoring. Sci. Rep. 5, 1-11 (2015).

29. Tsuji, Y., Sakai, H., Feng, L., Guo, X. \& Murata, H. Dual-gate low-voltage organic transistor for pressure sensing. Appl. Phys. Express 10, 021601 (2017).

30. Thuau, D. et al. Piezoelectric polymer gated OFET: Cutting-edge electro-mechanical transducer for organic MEMS-based sensors. Sci. Rep. 6, 38672 (2016).

31. Wu, N. et al. Theoretical study and structural optimization of a flexible piezoelectret-based pressure sensor. J. Mater. Chem. A 6, 5065-5070 (2018).

32. Liu, S. et al. Ultrasensitive $2 \mathrm{D} \mathrm{ZnO}$ Piezotronic Transistor Array for High Resolution Tactile Imaging. Adv. Mater. 29, 1606346 (2017).

33. Chang, C., Tran, V. H., Wang, J., Fuh, Y. K. \& Lin, L. Direct-write piezoelectric polymeric nanogenerator with high energy conversion efficiency. Nano Lett. 10, 726-731 (2010).

34. Park, K.-I. et al. Piezoelectric BaTiO3 thin film nanogenerator on plastic substrates. Nano Lett. 10, 4939-4943 (2010).

35. Lee, J. H. et al. Highly stretchable piezoelectric-pyroelectric hybrid nanogenerator. Adv. Mater. 26, 765-769 (2014).

36. Liu, S. et al. Ultrafast Dynamic Pressure Sensors Based on Graphene Hybrid Structure. ACS Appl. Mater. Interfaces 9, 24148-24154 (2017).

37. Chen, M. et al. An ultrahigh resolution pressure sensor based on percolative metal nanoparticle arrays. Nat. Commun. 10, 4024 (2019).

38. Kim, Y.-R. et al. Binary Spiky/Spherical Nanoparticle Films with Hierarchical Micro/Nanostructures for High-Performance Flexible Pressure Sensors. ACS Appl. Mater. Interfaces 12, 58403 (2020).

39. You, I. et al. E-Skin Tactile Sensor Matrix Pixelated by Position-Registered Conductive Microparticles Creating Pressure-Sensitive Selectors. Advanced Functional Materials vol. 28 1801858 (2018).

40. Zhu, G.-J. et al. A highly sensitive and broad-range pressure sensor based on polyurethane mesodome arrays embedded with silver nanowires. ACS Appl. Mater. Interfaces 12, 19988-19999 (2020).

41. Zhao, T. et al. Highly Sensitive Flexible Piezoresistive Pressure Sensor Developed Using Biomimetically Textured Porous Materials. ACS Appl. Mater. Interfaces 11, 29466-29473 (2019).

42. Fang, X., Tan, J., Gao, Y., Lu, Y. \& Xuan, F. High-performance wearable strain sensors based on fragmented carbonized melamine sponges for human motion detection. Nanoscale 9, 1794817956 (2017).

43. Song, Z. et al. Bioinspired Microstructured Pressure Sensor Based on a Janus Graphene Film for Monitoring Vital Signs and Cardiovascular Assessment. Adv. Electron. Mater. 4, 1800252 (2018). 
44. Tang, X. et al. Multilevel Microstructured Flexible Pressure Sensors with Ultrahigh Sensitivity and Ultrawide Pressure Range for Versatile Electronic Skins. Small 15, 1804559 (2019).

45. Lee, B. et al. Ultraflexible and transparent electroluminescent skin for real-time and superresolution imaging of pressure distribution. Nat. Commun. 11, 1-11 (2020).

46. Lee, Y. et al. Bioinspired Gradient Conductivity and Stiffness for Ultrasensitive Electronic Skins. ACS Nano 15, 1795-1804 (2021).

47. Choong, C.-L. et al. Highly stretchable resistive pressure sensors using a conductive elastomeric composite on a micropyramid array. Adv. Mater. 26, 3451-3458 (2014).

48. Huang, Z. et al. Pyramid microstructure with single walled carbon nanotubes for flexible and transparent micro-pressure sensor with ultra-high sensitivity. Sensors Actuators, A Phys. 266, 345351 (2017).

49. Li, H. et al. Ultrahigh-Sensitivity Piezoresistive Pressure Sensors for Detection of Tiny Pressure. ACS Appl. Mater. Interfaces 10, 20826-20834 (2018).

50. Yuan, L. et al. Synergistic Resistance Modulation toward Ultrahighly Sensitive Piezoresistive Pressure Sensors. Adv. Mater. Technol. 5, 1901084 (2020).

51. Li, G., Chen, D., Li, C., Liu, W. \& Liu, H. Engineered Microstructure Derived Hierarchical Deformation of Flexible Pressure Sensor Induces a Supersensitive Piezoresistive Property in Broad Pressure Range. Adv. Sci. 7, 2000154 (2020).

52. Jung, B. K. et al. Janus-like Jagged Structure with Nanocrystals for Self-Sorting Wearable Tactile Sensor. ACS Appl. Mater. Interfaces 13, 6394-6403 (2021).

53. Park, J. et al. Tactile-direction-sensitive and stretchable electronic skins based on human-skininspired interlocked microstructures. ACS Nano 8, 12020-12029 (2014).

54. Roh, E., Hwang, B. U., Kim, D., Kim, B. Y. \& Lee, N. E. Stretchable, Transparent, Ultrasensitive, and Patchable Strain Sensor for Human-Machine Interfaces Comprising a Nanohybrid of Carbon Nanotubes and Conductive Elastomers. ACS Nano 9, 6252-6261 (2015).

55. Liu, W. et al. A flexible and highly sensitive pressure sensor based on elastic carbon foam. $J$. Mater. Chem. C 6, 1451-1458 (2018).

56. Jin, S. et al. Low temperature polycrystalline silicon with single orientation on glass by blue laser annealing. Thin Solid Films 616, 838-841 (2016).

57. Kichambare, P. D. et al. Laser irradiation of carbon nanotubes. Mater. Chem. Phys. 72, 218-222 (2001).

58. Zhao, L. et al. Construction of sandwich-like porous structure of graphene-coated foam composites for ultrasensitive and flexible pressure sensors. Nanoscale 11, 10229-10238 (2019).

59. Zhang, X. et al. Flexible, Highly Sensitive, and Ultrafast Responsive Pressure Sensor with Stochastic Microstructures for Human Health Monitoring. Adv. Eng. Mater. 23, 1-9 (2021).

60. Joo, M. \& Lee, M. Laser treatment of solution-deposited carbon nanotube thin films for improved conductivity and transparency. Nanotechnology 22, 265709 (2011). 
61. Li, X., Hasan, M. M., Kim, H. M. \& Jang, J. Oxide Electronics Transferred on Stiff-Stripe/PDMS Substrate for High-Resolution Stretchable Displays. IEEE Trans. Electron Devices 66, 2971-2978 (2019).

62. Chen, Y. et al. An 18.6-\$ $\mu \$ \mathrm{~m}-$ Pitch Gate Driver Using a-IGZO TFTs for Ultrahigh-Definition AR/VR Displays. IEEE Trans. Electron Devices 67, 4929-4933 (2020).

63. Takei, K. et al. Nanowire active-matrix circuitry for low-voltage macroscale artificial skin. Nat. Mater. 9, 821-826 (2010).

64. Wang, C. et al. User-interactive electronic skin for instantaneous pressure visualization. Nat. Mater. 12, 899 (2013).

65. Yeom, C. et al. Large-area compliant tactile sensors using printed carbon nanotube active-matrix backplanes. Adv. Mater. 27, 1561-1566 (2015).

66. Lee, W. et al. OPEN A fully roll-to-roll gravure-printed carbon nanotube-based active matrix for multi-touch sensors. Nat. Publ. Gr. 1-10 doi:10.1038/srep17707.

67. Nela, L., Tang, J., Cao, Q., Tulevski, G. \& Han, S.-J. Large-Area High-Performance Flexible Pressure Sensor with Carbon Nanotube Active Matrix for Electronic Skin. Nano Lett. 18, 2054 2059 (2018).

68. Someya, T. et al. A large-area, flexible pressure sensor matrix with organic field-effect transistors for artificial skin applications. Proc. Natl. Acad. Sci. 101, 9966-9970 (2004).

69. Someya, T. et al. Conformable, flexible, large-area networks of pressure and thermal sensors with organic transistor active matrixes. Proc. Natl. Acad. Sci. 102, 12321-12325 (2005).

70. Noguchi, Y., Sekitani, T. \& Someya, T. Organic-transistor-based flexible pressure sensors using ink-jet-printed electrodes and gate dielectric layers. Appl. Phys. Lett. 89, 253507 (2006).

71. Zang, Y. et al. Flexible suspended gate organic thin-film transistors for ultra-sensitive pressure detection. Nat. Commun. 6, 6269 (2015).

72. Mativenga, M., Geng, D., Kim, B. \& Jang, J. Fully transparent and rollable electronics. ACS Appl. Mater. Interfaces 7, 1578-1585 (2015).

73. Kim, Y.-H., Lee, E., Um, J. G., Mativenga, M. \& Jang, J. Highly robust neutral plane oxide TFTs withstanding $0.25 \mathrm{~mm}$ bending radius for stretchable electronics. Sci. Rep. 6, 25734 (2016).

\section{Acknowledgements}

This work was supported by the National Research Foundation of Korea (NRF) grant funded by the Korea government (MSIT) (No. 2020M3H4A1A02084899).

\section{Author contributions}

All authors contributed to this work equally.

\section{Competing interests}

The authors declare no competing financial interests. 


\section{Data availability}

The authors confirm that all the data supporting the findings of this study are available within the article [and/or] its supplementary materials. 


\section{Supplementary Files}

This is a list of supplementary files associated with this preprint. Click to download.

- CJParkSRSupporting.pdf 